\title{
Identifying Real-world Credible Experts in the Financial Domain
}

\author{
TENG-CHIEH HUANG, RAZIEH NOKHBEH ZAEEM, and K. SUZANNE BARBER, \\ The University of Texas at Austin, USA
}

\begin{abstract}
Establishing a solid mechanism for finding credible and trustworthy people in online social networks is an important first step to avoid useless, misleading, or even malicious information. There is a body of existing work studying trustworthiness of social media users and finding credible sources in specific target domains. However, most of the related work lacks the connection between the credibility in the real-world and credibility on the Internet, which makes the formation of social media credibility and trustworthiness incomplete. In this article, working in the financial domain, we identify attributes that can distinguish credible users on the Internet who are indeed trustworthy experts in the real-world. To ensure objectivity, we gather the list of credible financial experts from real-world financial authorities. We analyze the distribution of attributes of about $10 \mathrm{~K}$ stock-related Twitter users and their 600K tweets over six months in 2015/2016, and over 2.6M typical Twitter users and their $4.8 \mathrm{M}$ tweets on November 2nd, 2015, comprising $1 \%$ of the entire Twitter in that time period. By using the random forest classifier, we find which attributes are related to real-world expertise. Our work sheds light on the properties of trustworthy users and paves the way for their automatic identification.
\end{abstract}

\author{
CCS Concepts: • Networks $\rightarrow$ Social media networks; • Information systems $\rightarrow$ Relevance assessment; \\ Additional Key Words and Phrases: Source credibility, trust, financial market
}

\section{ACM Reference format:}

Teng-Chieh Huang, Razieh Nokhbeh Zaeem, and K. Suzanne Barber. 2021. Identifying Real-world Credible Experts in the Financial Domain. Digit. Threat.: Res. Pract. 2, 2, Article 17 (April 2021), 14 pages.

https://doi.org/10.1145/3446783

\section{INTRODUCTION}

For most of the human history, trust among people has been judged through the physical world such as in-person communication, letters, or phone calls. The invention of the Internet and the emergence of social networks, however, has thoroughly altered the way people communicate. In the blink of an eye, everyone can reach almost every corner of the world through online social networks. The quantity of sources a person can contact has grown enormously, while some of these sources may not deserve one's trust and might deceive him/her. This research seeks to answer an ever-pressing question for those who rely on social media for information: Who can I trust? Our contribution is to focus on the connection between trustworthiness and credibility of a user in the real-world and that user's credibility on the Internet.

Authors' addresses: T.-C. Huang, R. N. Zaeem, and K. S. Barber, The University of Texas at Austin, Center for Identity, 2501 Speedway, MS C4300, Austin, TX 78712; emails: tengchieh@utexas.edu, \{razieh, sbarber\}@identity.utexas.edu.

Permission to make digital or hard copies of all or part of this work for personal or classroom use is granted without fee provided that copies are not made or distributed for profit or commercial advantage and that copies bear this notice and the full citation on the first page. Copyrights for components of this work owned by others than ACM must be honored. Abstracting with credit is permitted. To copy otherwise, or republish, to post on servers or to redistribute to lists, requires prior specific permission and/or a fee. Request permissions from permissions@acm.org.

(c) 2021 Association for Computing Machinery.

2576-5337/2021/04-ART17 \$15.00

https://doi.org/10.1145/3446783

Digital Threats: Research and Practice, Vol. 2, No. 2, Article 17. Publication date: April 2021. 
By connecting the traditional definition of trust (e.g., reputation, expertise toward certain professional fields, and experience related to the target domain) to many personal attributes retrieved from social networks (such as user information and social interconnection), we establish a dependable way to recognize trustworthy users. This work aims to thoroughly investigate which user attributes could be retrieved as a differentiation tool for the degree of credibility.

To automatically identify social media users who are in fact credible experts in the real-world, we study social media attributes of a set of actual real-world experts. We focus on the financial market as the target domain. To begin with, we referenced some of the most trustworthy and prestigious journalists or media sources. In this research, the financial experts are suggested by articles of these trustworthy sources. Combined with typical users and stock-related users, we are able to compare the differences among these groups.

We leverage the concept of trust filter scores-measures that evaluate different aspects of user credibility. We hope using actual financial experts in the real-world and their Twitter feed and the study of their trust filter scores can help us explain why some user attributes are better indicators of credibility-i.e., some trust filters perform better than others. We also aim to understand which trust filters are more suitable for specific target domains, which in our study is the financial market. We therefore analyze user attributes extracted from social networks and connect them with trust filters. Furthermore, by analyzing the distribution of user attributes among various groups, we achieve a better estimation of the importance of different attributes.

This article makes the following contribution: We select testing groups based on objective and neutral thirdparty sources and hence provide an unbiased comparison of the user attributes. Instead of relying on self-defined or machine learning methods to identify credibility, this research provides an alternative way of understanding the differences between the experts and others.

The remaining of this article is organized as follows: Section 2 reviews related literature including prediction by analyzing social media data, the history of the trust filter, and the definition of trust and credibility. Section 3 specifies how we retrieve data and select attributes. Section 4 presents our results based on different user attributes versus types of social media users. Section 5 concludes the article and suggests some possible avenues for future work.

\section{RELATED WORK}

Given the sheer amount of research performed on social media data, in this section, we seek to review the most closely related work to place our work and highlight its differences and contributions.

\subsection{The Credibility of Social Media Data}

Any research or practical application (including those in the financial market domain [15]) that retrieves data from social media sources has to face a major concern: Some social media data might be unreliable, untrustworthy, or even malicious. Some of the most significant properties of social media (from the fast spread of information and the low cost and effort of broadcasting news to the ease of hiding one's identity) now become a nest for fake news. Some researchers $[1,30]$ have studied the spread of fake news on social media during election seasons. Many $[6,10,33]$ have applied data mining and machine learning techniques to detect fake news on social media. Some [17] have performed rumor detection on social media based on various data mining algorithms such as decision trees, random forest, and SVM. More and more, researchers consider the credibility problem in social media as a major concern $[1,9,23,25,28,29,33,37]$.

\subsection{Social Media User Credibility}

A body of work $[9,12,21]$ exists that particularly looks at the credibility of Twitter users. For example, Castillo et al. [5] used a cascade of machine learning models (classifiers) to first find newsworthy and then identify credible tweets. In another work [4], they discovered that credible news is propagated through authors who

Digital Threats: Research and Practice, Vol. 2, No. 2, Article 17. Publication date: April 2021. 
write a large number of posts, originate at a single or few users in the network, and have many re-tweets. Since surely fake news can spread in a similar manner, they also point out that tweets that do not include URLs tend to be related to non-credible news, those with negative sentiment tend to be more credible, and those with question marks or smiling emoticons are more likely to spread non-credible information. Gupta et al. [11] found that only $17 \%$ of the dataset they considered contained credible situational awareness information. They used regression analysis to identify two sets of relevant features, namely, content-based features (e.g., the number of unique characters or emoticons in a tweet) and user-based features (e.g., the number of followers or length of username). They suggested the use of those features as credibility scores. Canini et al. [3] performed a similar credibility ranking. Our work takes into account the features suggested by similar papers $[4,13,27]$.

\subsection{Application of Classifiers in Detecting Credible Users}

Researchers [16, 26, 34] have applied machine learning classifiers, such as random forest, to detect credible news sources on social media. Our contribution lies in our way of enlisting credible users and in the observation of the value distribution for features and not in the application of machine learning classifiers.

\subsection{Trust Filters}

The term trust filters was first introduced in Reference [19], where the original idea was to filter and rank trustworthy social media users. There were six trust filters: Authority, Experience, Expertise, Identity, Proximity, and Reputation. All six filters stem from the traditional way of estimating one's trustworthiness. After that, an Internet bio-surveillance application [36] was developed to investigate and detect possible outbreak of diseases in early stages.

Even though there are fundamental differences between user trust and credibility, we use trust to mean credibility in this work. User trust is the subjective expectation of a user of the other; credibility is an objective description of the level of trustworthiness that one possesses. Some surveys [2, 32] have covered conceptual differences between trust and credibility. A previous work [14] merges the objective credibility and subjective trust to make them interchangeable in terms of meaning. Furthermore, it applied this concept as a reference to a specific target domain of stock markets. By doing so, it achieved a more accurate prediction of stock prices based on Twitter sentiment analysis by leveraging trust filters.

\section{METHODOLOGY}

This section explains how we extract social media data, decide which attributes to include, and how many experimental groups of users to use. The social media we utilize is Twitter. Given its property of short posts, we think Twitter would be a great fit for the fast-changing financial market. Moreover, we could access more posts and users with our available computational power and data capacity, which would be beneficial to this work when analyzing the distribution of social media users. We derive a set of attributes for each user and use the value of those attributes to judge the trustworthiness of users. Here, an attribute refers to a user, text, or social connection information of a single social media user. We comprehensively reviewed previous work [4, 13, 27] and selected a set of attributes for this research. We analyze the distribution of those attribute values for users and apply data-mining techniques, such as random forest, to evaluate trustworthiness and the best attributes to quantify it. The final goal is understanding how important any attribute is when establishing an individual's trustworthiness on social media.

\subsection{Attribute Selection}

We chose 11 attributes (shown in Table 1). These attributes are based on previous research [4, 13, 27] and use tweets content, Twitter user information, or social network structure. Both References [13] and [4] aim to verify the information credibility of tweets, and Reference [27] focuses on the correlation between tweets and the 
Table 1. Attributes Extracted from Tweets

\begin{tabular}{ll}
\hline Time-independent Attributes & \multicolumn{1}{c}{ Definition } \\
\hline n_tweet & Number of tweets collected in dataset \\
stock_related_tweet & Number of tweets that contain a stock symbol \\
statuses_count & Total number of posted tweets in user history \\
followers_count & Number of followers of a user \\
friends_count & Number of friends (following users) of a user \\
avg_len_tweet & Average tweet length in characters per tweet \\
avg_n_word_tweet & Average number of words per tweet \\
avg_hashtag & Average number of hashtag symbols per tweet \\
avg_tweet_URL & Proportion of tweet containing URLs \\
avg_tweet_question & Proportion of tweet containing “?”" \\
avg_tweet_exclamation & Proportion of tweet containing "!" \\
\hline
\end{tabular}

financial domain. All of these papers compare a variety of Twitter attributes and list the most promising attributes based on data-mining algorithms such as decision tree classifier or SVM. Ruiz et al. [27] suggest the statuses_count is one of the most highly related features for the stock market. Gupta et al. [13] conclude that followers_count, statuses_count, avg_len_tweet, avg_tweet_URL, avg_tweet_exclamation, and avg_hashtag are top features for evaluating event credibility. Finally, Castillo et al. [4] found avg_tweet_URL, avg_tweet_exclamation, statuses_count, and friends_count as the most important features for identifying the credibility of news. We consider all the above recommended attributes, plus stock_related_tweet, which is highly relevant to our topic. We chose attributes that are neutral and suitable for universal purposes across domains and do not require any specialized retrieval technique to calculate the attribute value for a user. These attributes can be directly applied to various different target domains such as politics or entertainment. Even in the case of stock_related_tweet, we can simply switch the keywords to fit various target domains.

As a part of future work, we plan to include other well studied features from the literature [10, 24].

\subsection{User Group Categorization}

We retrieve data from a publicly available collection of tweets for the Spritzer version from Internet Archive, which is a non-profit digital library. The Spritzer version contains approximately a $1 \%$ sample of Twitter public posts. The sampled data is examined and shown to preserve enough information for the research and application based on the tweet or content statistics [35]. We use the entire Spritzer data. Our data sampling time period was from November 2015 to April 2016. We analyze the distribution of attributes of 9,324 stock-related Twitter users and their 593,344 tweets over six months in 2015/2016, and 2,655,283 typical Twitter users and their 4,811,258 tweets on November 2nd, 2015, comprising $1 \%$ of the entire Twitter in those time periods.

We consider three groups of users:

(1) Typical users, who represent a random sampling of all Twitter users. This group stands for the baseline among all groups. Typical users serve as the control group and represent how the social media community should look like, so we can distinguish if there exists a distinct distribution of attributes in the other groups. Therefore, there should be no condition of selecting users except the sampling time.

(2) Stock-related users are those who post at least one tweet with a reference to a stock market symbol during the sampling time period. With this group, we aim to represent the users who might be interested in the stock market, while they may not be experts. Therefore, this group can help us differentiate between true financial experts and ordinary people with financial interest. In this work, we only focus on tweets containing the symbols of the stock market companies, e.g., \$AAPL or \$TSLA. The stock-related tweet

Digital Threats: Research and Practice, Vol. 2, No. 2, Article 17. Publication date: April 2021. 
collections do not include tweets only mentioning the company name. One rationale is to prevent wrongly collecting unrelated tweets, for example those that refer to "apple" as a fruit or "Tesla" as a scientist.

(3) Financial expert users are retrieved from well-known online sources (Business Insider's article titled "The 129 finance people you have to follow on Twitter" [20]; an article from CommodityHQ.com titled "100 Insightful Futures Traders Worth Following on Twitter" [8], which is also quoted by NASDAQ.com; and Forbes' "Must-Follow Twitter Feeds on Markets And Economics" [7]). These lists are compiled by their authors or by Wall Street analysts and journalists, who are traditionally considered financial authorities. Business Insider's article asked Wall Street analysts and Business Insider journalists to list their must-follow tweeters. CommodityHQ.com selected experts based on various criteria: activeness, knowledge, excellent investors, experience, expertise on a particular sector or asset, and whether the account is directly run by the experts. For the article in Forbes, the list is suggested by the author himself. Therefore, the expert list is based on multiple aspects and is not just limited to a single source. Since our Twitter sampling time period is in 2015 and 2016, we only referenced articles posted between 2015 and 2016. Originally, there were 257 people/groups mentioned in these articles. However, we were unable to find some accounts, since they might have been deactivated by Twitter or their owner. After manually examining all the accounts, we kept 228 of them.

We sampled data from November 2015 to April 2016. However, given the sheer amount of data, this time period was not suitable for the general users group, as it would result in a huge dataset we could not effectively examine (682,271,432 tweets posted by 63,873,729 users, which contributed to 2.1 TB amount of data). Hence, the time interval we selected for typical users was November 2, 2015. For November 2, there are still 4,811,258 tweets posted by 2,655,283 users. The number of posts and users from November 2 is approximately of the same magnitude of the other two groups over the six-month period. A caveat is that some attributes would no longer be applicable if we have different time periods for different user types. Take n_tweet for example. To still use such attributes, we multiply the value of such attributes for typical users from November 2 by 183 (the total number of days from November 2015 to April 2016) to roughly put two groups of users with different time intervals into comparison. Nevertheless, the number of tweets a user posted in a single day does not always stay the same; on the contrary, it usually varies a lot. We will cover more about this problem in Section 4. We reiterate that we monitored the stock-related users and financial experts over the entire six months.

\section{RESULTS}

This section presents and discusses our results. To better compare data with different sizes of expert groups, we have normalized the charts. The Y-axis shows the probability density of users instead of the absolute number throughout this section. Because the time frame for typical users is November 2, 2015, normalizing the time axis is also necessary for time-sensitive attributes such as the number of tweets (n_tweet) and the number of tweets that contain a stock symbol (stock_related_tweet). The line charts were transformed from the interpolation of bar charts. For the balance of accuracy and readability, we set a bin number of $20 \mathrm{for}$ all bar charts except the distribution of the average number of words per tweet (Figure 7), which is set to 30 . This is because only the assigned range for Figure 7 is not divisible by 20 and would cause zigzag shape distribution in the line chart (Figure 8).

\subsection{Attribute Distribution Analysis}

We found from Figure 1 and Figure 2 that the distribution of typical users and stock-related users is almost the same. Experts show a relatively higher proportion of prolific writers in terms of $\mathrm{n}_{-}$tweet and stock-related tweets.

Figure 3 shows the status count, i.e., the number of tweets a user has ever posted. It shows a similar trend as Figure 1 and Figure 2: There is no meaningful difference between typical users and stock-related users. However, a bigger percentage of financial experts have posted between 25,000 and 125,000 times.

Digital Threats: Research and Practice, Vol. 2, No. 2, Article 17. Publication date: April 2021. 


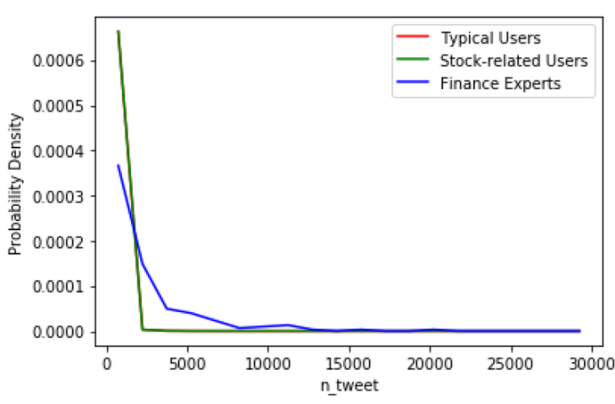

Fig. 1. The distribution of $\mathrm{n}_{-}$tweet for the three user groups. Typical users and stock-related users are almost identical and cannot be differentiated.

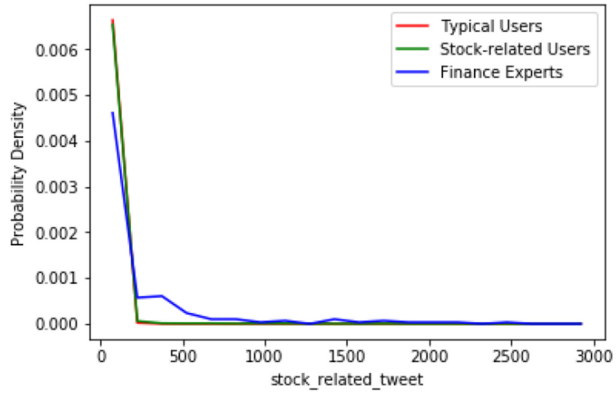

Fig. 2. The distribution of stock_related_tweet. Typical users and stock-related users are almost identical and cannot be differentiated.

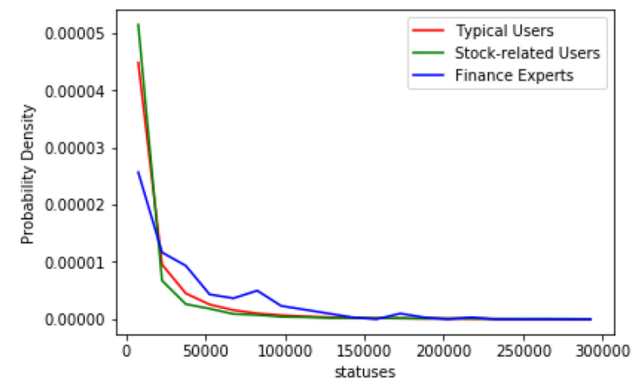

Fig. 3. The distribution of statuses_count.

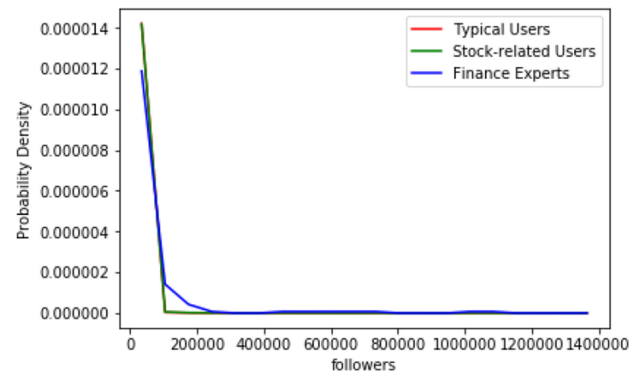

Fig. 4. The distribution of the number of followers.

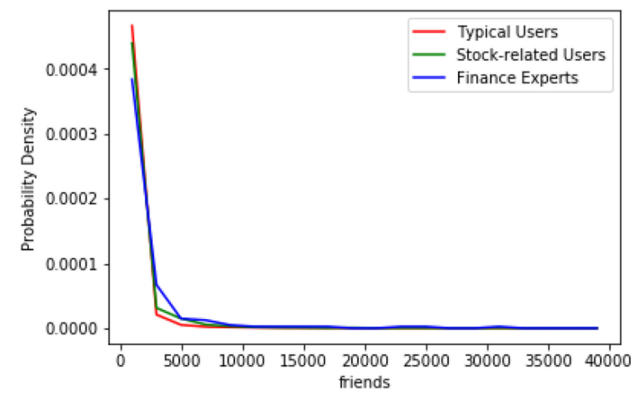

Fig. 5. The distribution of following users.

Figure 4 and Figure 5 are followers count and following (friends) count, respectively. The experts in followers count have a much higher proportion of large follower users, which surpasses the other two groups. This trend sustains while not so overwhelming in the following count. Compared to the experts, the difference between typical users and stock-related users is not so obvious.

The distribution of avg_len_tweet for stock-related users and experts is similar (Figure 6), which both have peaks at around 110 characters. The typical users, however, have a very different distribution for their average tweet length. Consequently, the average tweet length is an attribute that can distinguish between typical users

Digital Threats: Research and Practice, Vol. 2, No. 2, Article 17. Publication date: April 2021. 


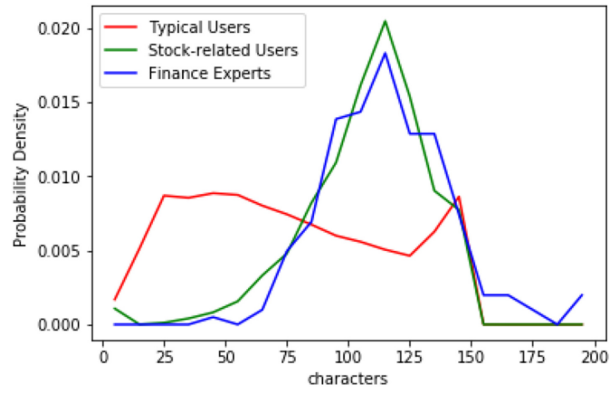

Fig. 6. The distribution of the average number of characters per tweet.

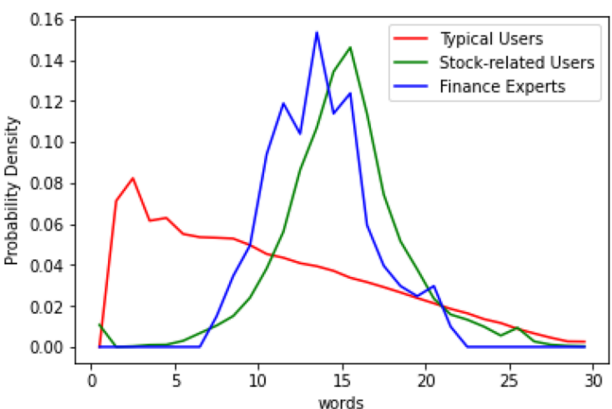

Fig. 7. The distribution of the average number of words per tweet.

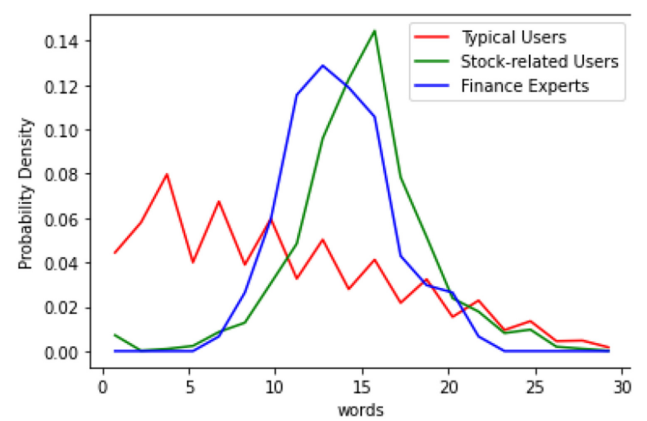

Fig. 8. The distribution of the average number of words per tweet when the number of bins is 20 .

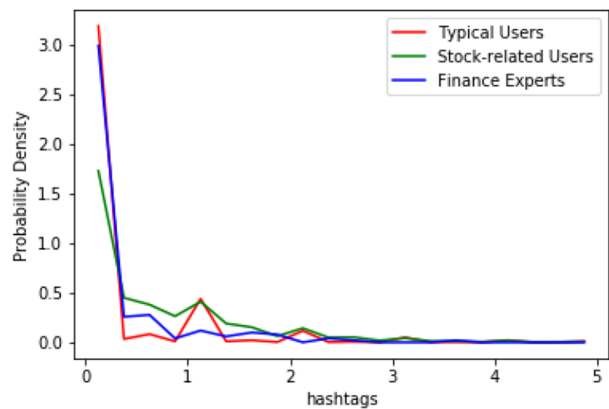

Fig. 9. The distribution of the average number of hashtags per tweet.

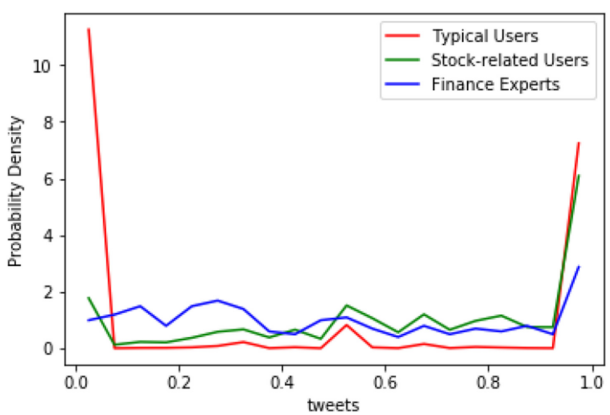

Fig. 10. The distribution of the proportion of tweets containing URLs.

and experts or people with an interest in the stock market. Similarly, the distribution of avg_n_word_tweet in Figure 7 peaks around 15 words for stock-related users and experts.

For avg_hashtag in Figure 9, there are some peaks for typical users, while the percentage of users with a given average number of hashtags per tweet decreases steadily for stock-related users and experts. We find that there exists many users who only post one or two tweets and make the integer numbers in the distribution distinct.

As for avg_tweet_URL in Figure 10, stock-related users and experts have a greater proportion of users between 0.1 to 0.9 compared to typical users. 


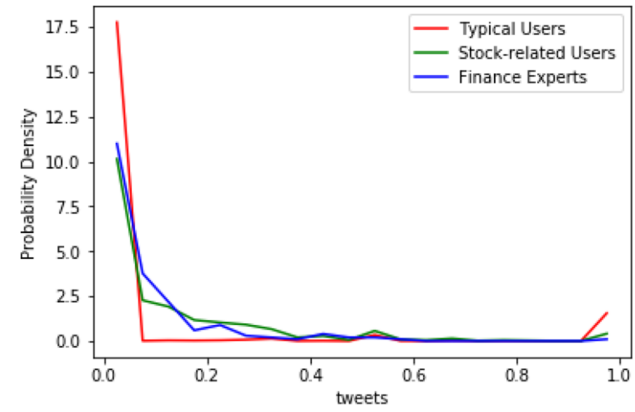

Fig. 11. The distribution of the proportion of tweets containing "!".

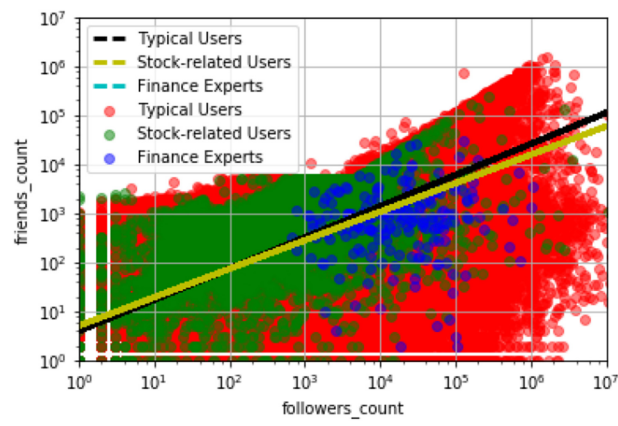

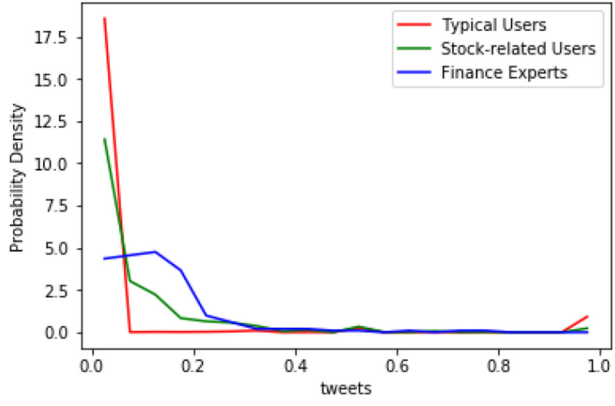

Fig. 12. The distribution of proportion of tweets containing "?".

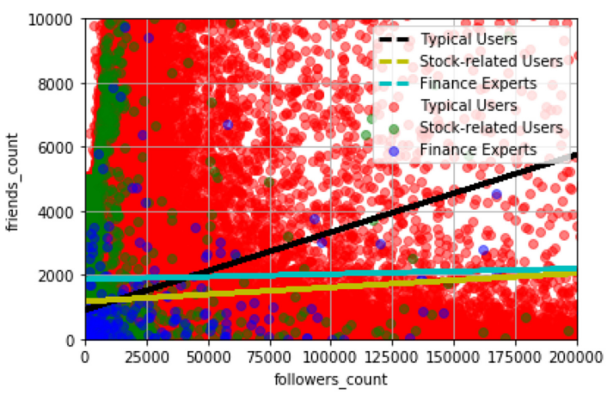

Fig. 13. (Left) The distribution of users in terms of friends_count and followers_count. Each dot represents one user. (Right) The distribution set with a smaller focus region. There are three trendlines representing the three user groups, respectively. Slope for each trendline: Typical: 0.0243, Stock-related: 0.0044, Expert: 0.0017 .

Figure 11 and Figure 12 show the number of users with a given average number of exclamation points and question marks per tweet. Stock-related users and experts have similar distributions for exclamation points, and both of their distributions are mostly higher than the one for typical users. The distribution of avg_tweet_question in experts is higher than the distribution of stock-related users for values larger than 0 , while both of them are much higher than the distribution of typical users. We can see that typical users have a peak when avg_tweet_URL, avg_tweet_exclamation and avg_tweet_question equal 1 (Figure 10, Figure 11, and Figure 12). This happens because of the higher ratio of typical users composing merely one tweet in our entire sampling. If the one tweet for such users contains a URL, an exclamation point, or a question mark, the average number will equal one.

\subsection{Correlation Between Attribute Pairs}

Based on the above results, to better understand the correlation between attributes and the testing groups, we chose some attribute pairs to investigate the distribution of the Twitter users. Those attribute pairs are (1) followers_count vs. friends_count; (2) avg_len_tweet vs. avg_n_word_retweet; and (3) n_tweet vs. stock_related_tweet.

Figure 13 shows the distribution of each group in terms of followers count and friends count. There is a clear boundary in Figure 13 (left) because of two Twitter follower regulations: (1) The 5,000 friends limit: If a user has less than 5,000 followers, then the maximum he/she can follow is 5,000 accounts. (2) The $10 \%$ limit: If a user follows more than 5,000 users, then it would be limited to $10 \%$ more than the number of people that follow the user. For example, if 5,000 people follow user X, then X can follow a max of 5,500 people; if 10,000 people follow user Y, then Y can follow up to 11,000 people, and so on. Figure 13(right) displays the same results in a smaller 


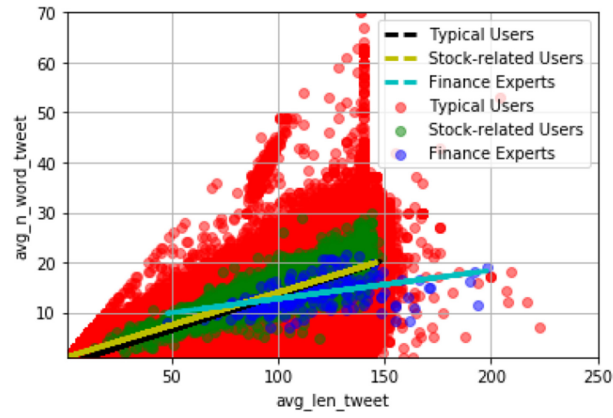

Fig. 14. The distribution of users in terms of avg_len_tweet and avg_n_word_tweet. Each dot represents one user. There are three trendlines representing the three user groups, respectively. Slope for each trendline: Typical: 0.14, Stock-related: 0.13, Expert: 0.056 .

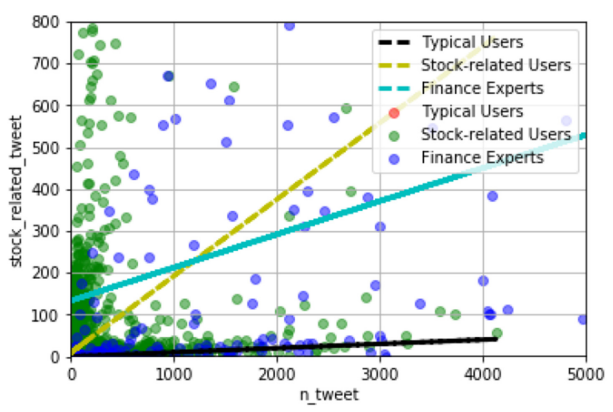

Fig. 15. The distribution of users in terms of n_tweet and stock_related_tweet. Each dot represents one user. Because the quantity of n_tweet and stock_related_tweet in typical users are much smaller than stock-related users and experts, the red spots are hardly seen in this figure. However, we can still see there are three trendlines representing the three user groups, respectively. Slope for each trendline: Typical: 0.01, Stock-related: 0.183, Expert: 0.079 .

focus region where a set of trendlines for each group is depicted. The slope of the trendline for financial experts is smaller than the other two groups. This new attribute could also be an identifiable characteristic for various users.

When comparing avg_n_word_tweet versus avg_len_tweet (Figure 14), the slope of the trendline for finance experts is quite different than the other two, which are steeper. This means that the experts tend to use less words for a given length of tweet, i.e., they use longer and more sophisticated words.

Comparing n_tweet and stock_related_tweet (Figure 15), the slope of the trendline for finance experts is higher than typical users, but smaller than stock_related users. It is reasonable, because the definition of a stock_related user is whoever contributed any stock_related tweet. Considering that, finance experts do tend to contribute much more stock-related tweets than regular users.

\subsection{Random Forest Analysis}

The random forest algorithm [18] has been broadly applied on classification and regression tasks. Random forest is a technique combining decision tree, bagging, and random selection of features. One of the benefits of random forest is it can rank each variable according to its importance, which can help us understand how suitable the set of attributes is for the target domain. Random forest also can handle large quantities of data very well, since it can be implemented with parallelism, and it can maintain fast prediction and training speed.

Table 2 shows the results using random forest ${ }^{1}$ models to distinguish each group: typical users from experts in the second column, stock-related users from experts in the third column, and all three groups from each other in the last column. We consider only time-independent attributes, because the sampling time lengths are different among the three groups. Therefore, there are only nine attributes listed in Table 2.

We randomly selected $10 \%$ of all the users as the test set and utilized the other users as the training set. After testing different parameters from 5 to 500 (Figure 16, where accuracy and AUC do not increase significantly when number of trees surpasses 50), we set the number of trees to 50 for the balance of speed and performance.

\footnotetext{
${ }^{1}$ We used the Scikit-learn library.
} 
Table 2. Machine Learning Feature Importance of Each Attribute

\begin{tabular}{lccc}
\hline Time-independent Attributes & Typical Users \& Expert & Stock_Related Users \& Experts & All \\
\hline statuses_count & $\underline{0.0621}$ & $\underline{0.0687}$ & $\underline{0.0730}$ \\
followers & 0.0863 & $\mathbf{0 . 2 2 8 5}$ & 0.1208 \\
friends_count & 0.0823 & $\underline{0.0689}$ & $\underline{0.0848}$ \\
avg_len_tweet & 0.0869 & $\mathbf{0 . 2 0 2 7}$ & $\mathbf{0 . 1 3 1 9}$ \\
avg_n_word_tweet & $\underline{0.0460}$ & 0.0809 & $\underline{0.0720}$ \\
avg_hashtag & $\mathbf{0 . 1 7 2 6}$ & 0.0785 & $\mathbf{0 . 1 4 0 9}$ \\
avg_tweet_URL & $\underline{0.0710}$ & $\mathbf{0 . 1 1 2 4}$ & 0.1071 \\
avg_tweet_question & $\mathbf{0 . 1 3 5 1}$ & 0.0780 & 0.1029 \\
avg_tweet_exclamation & $\mathbf{0 . 2 5 7 7}$ & 0.0814 & $\mathbf{0 . 1 6 6 4}$ \\
\hline Accuracy & 0.999969 & 0.994524 & 0.999945 \\
AUC & 0.789474 & 0.821429 & 0.631579 \\
\hline
\end{tabular}

There are three comparison groups: Typical users \& Experts, Stock_related users \& Experts and all three compared together. Highest/lowest values of importance in each column are shown in bold/underlined, respectively. Prediction accuracy and AUC are also shown in the last two rows.
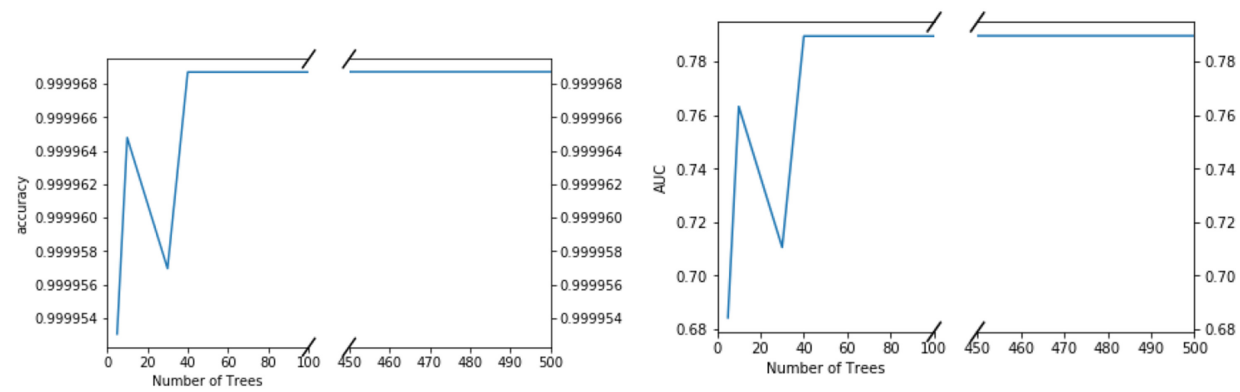

Fig. 16. (Left) Accuracy with different number of trees and (Right) AUC with different number of trees for the random forest algorithm.

Beside the accuracy and AUC (Area Under the Curve of Receiver Operating Characteristic curve), the feature importance is also shown in Table 2. AUC provides an aggregate measure of performance across all possible classification thresholds. It measures how well predictions are ranked, rather than their absolute values. This helps us understand which attributes are more influential during the comparison process. When comparing between two groups (typical users \& experts, stock-related users \& experts), the importance values have larger variation (range from 0.0460 to 0.2577 and from 0.0687 to 0.2285 ) and the top three most important attributes $(0.2577,0.1726$, and 0.1351 in the second column, and $0.2285,0.2027$ and 0.1124 in the third column) are totally distinct among the two comparing sets. However, when taking all three groups into account, the importance values become more evenly distributed (ranging from 0.0720 to 0.1664 ) and there is no particularly overwhelming attribute. We also observe that even the least-important attribute still cannot be ignored, which implies the entirety of the set of attributes we selected are mostly effective.

There are three attributes that frequently have the lowest importance values: statuses_count, friends_count, and avg_n_word_tweet. The former two are easier to manipulate, since users can post as many tweets as they wish or follow as many friends as they want, except for the friends limit mentioned above. These kinds of attributes are more unidirectional and do not require feedback from others. If a user wants to improve his or her trustworthiness on the social network, then it is also intuitive that the user would post a lot or eagerly extend their social connection. As a result, statuses_count and friends_count become relatively non-ideal attributes 


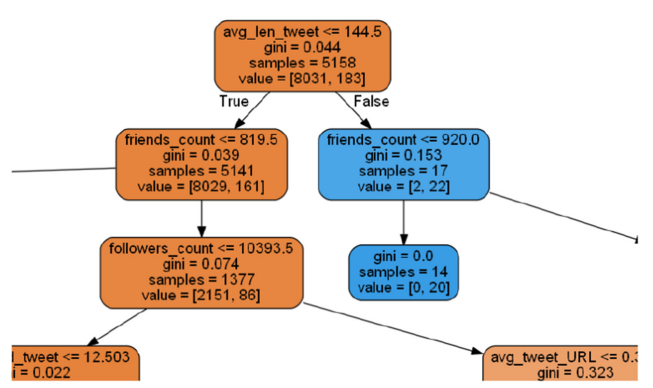

Fig. 17. Top portion of the decision tree 0 .

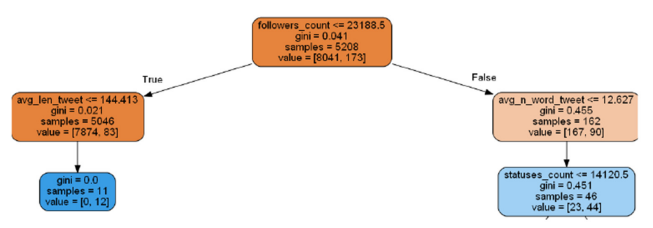

Fig. 19. Top portion of the decision tree25.

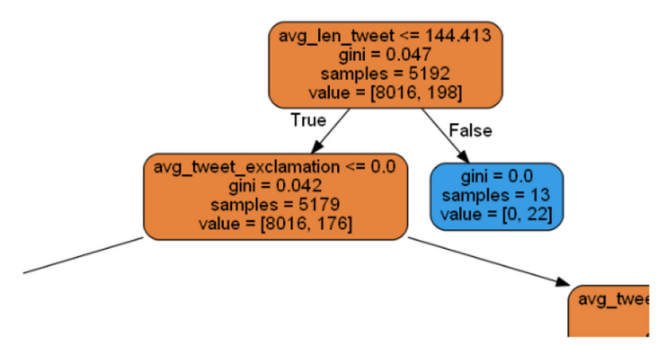

Fig. 18. Top portion of the decision tree15.

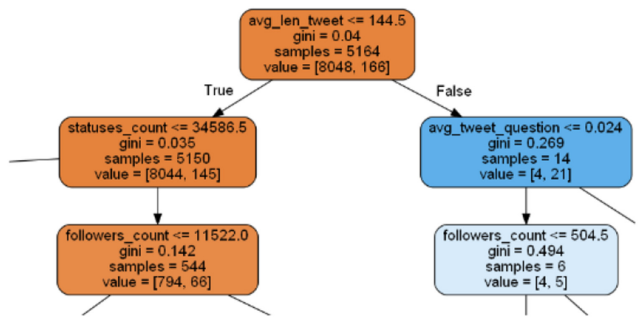

Fig. 20. Top portion of the decision tree35.

while classifying user groups. As for avg_n_word_tweet, it might be affected by avg_len_tweet, which possesses similar values among users.

However, when it comes to attributes with highest importance values, it is surprising the top attribute, avg_tweet_exclamation, is related to writing style. Our data shows that experts tend to restrain themselves from revealing too much expression in the words. Other than that, we notice attributes relevant to text content are mostly within higher importance values. This implies even without much social network connection, we could still distinguish experts from others by simply investigating their writing style.

Another intriguing phenomenon is when it comes to distinguishing stock-related users and experts, the second highest attribute in terms of important value is avg_len_tweet. However, from Figure 6, we found that the distribution of average number of characters per tweet (i.e., avg_len_tweet) for stock-related users and financial experts are almost the same around their peaks. In view of this, how can avg_len_tweet be considered as an important feature? Our speculation is that considering the extremely low percentage of financial experts among all users, the decision condition for the decision trees can be reasonably deduced that only very few users may qualify as financial experts. It is possible that avg_len_tweet is only being used to filter out a small portion of experts (for example, finding experts whose avg_len_tweet are over 150 characters) but can effectively separate some experts from others. To further support the assumption, we extract some decision trees from the random forest and plot the visualized graphs (Figures 17 to 20). We can observe that for all these trees there always exist avg_len_tweet on the level 1 or level 2 decision condition.

\subsection{Threats to Validity}

Internal Validity: Because the typical users are selected randomly through the sampling mechanism of the Spritzer database, there might exist experts in the typical user group. Our assumption was that the proportion of experts would be negligible in the typical users group. Therefore, the influence of the existence of experts in typical user distribution and in the application of random forest is limited and negligible. To verify this assumption, we split the typical users and reserve 100,000 of 2,655,385 users as "untouched data" for verification. 
Using the random forest prediction model, we found only 3 of 100,000 typical users are determined as experts. This very small number of experts would not interfere with the final results. In addition, there are 252 detected experts among the 9,425 stock-related users, which contributes to $2.67 \%$ of stock-related users.

External Validity: The most important threat to external validity is our identification of experts. To address this threat, we selected the list of experts from some of the most prestigious third-party independent media (from the Business Insider [20]; from CommodityHQ.com, which is also quoted by NASDAQ.com [8]; and from Forbes [7]), which means only the experts who possess both highly financial expertise and national or even global influence can be seen on the list. It is expected that only few people are qualified among tens of thousands of people, which is consistent with the number of experts we found in the typical user group.

Another threat to the external validity of this work is the selection of attributes, as we manually picked the attributes and designed the random forest model to evaluate them. It is important to make sure that only financial experts, not even experts from other fields, are correctly recognized by using these attributes. Although the features selected for random forest classifier are non-financial-related, it is still possible to preserve the writing style that is financial-specific. From the comparison of typical users and financial experts in Table 2, we can observe that the most important features are those related to the writing style (avg_hashtag, avg_tweet_question, and avg_tweet_exclamation). To further address this threat, we intend to select a more diverse set of attributes, other models, and more datasets. We have performed a preliminary literature review of appropriate attributes (e.g., References [10, 24]), models (e.g., References [6, 10,33]), and datasets (e.g., Reference [31]) for this future work. Finally, various different social media platforms [22] should be explored to see if these results from Twitter are applicable to them.

\section{CONCLUSION}

This research presented a distinctly novel view to differentiate credible and trustworthy users on social media. We referenced some of the most prestigious media listings of credible users instead of self-identifying them. We further utilized those listings to study the distribution of user attributes as well as for the training phase of classification methods. We focused on the financial market and $1 \%$ of all public tweets in a six-month period from Twitter as the target expertise domain and target social media, respectively. We categorized social media users in three different groups: typical, stock-related, and expert users, based on their relevant expertise on the financial market. We considered a set of user attributes based on our extensive review of related work to analyze the attribute distribution of each group. The experimental results show that based on attribute value distribution, some attributes are better indicators of expertise, such as the average length of tweets, the average number of words per tweet, and the number of followers, which display different distribution patterns in experts/stock-related users than typical users. We further applied random forest classification to enhance verification of this observation. We found that the frequency of using exclamation points (!) and hashtags (\#) are strong differentiators of experts from typical users and the number of followers and the average tweet length are best indicators for identifying stock-related users from experts using random forest classification. In addition, even among those users with high involvement in certain expertise domains (e.g., financial market in this research), our application of random forest classification can still identify true experts from merely interested users by considering multiple user attributes. Our work paves the way for analyzing news source credibility and expertise on social media. As part of future work, we plan to incorporate other attributes, models, datasets, and social media platforms.

\section{REFERENCES}

[1] Hunt Allcott and Matthew Gentzkow. 2017. Social media and fake news in the 2016 election. f. Econ. Perspect. 31, 2 (2017), 211-36.

[2] Majed Alrubaian, Muhammad Al-Qurishi, Atif Alamri, Mabrook Al-Rakhami, Mohammad Mehedi Hassan, and Giancarlo Fortino. 2018. Credibility in online social networks: A survey. IEEE Access 7 (Dec. 2018), 2828-2855. DOI : https://doi.org/10.1109/access.2018.2886314

Digital Threats: Research and Practice, Vol. 2, No. 2, Article 17. Publication date: April 2021. 
[3] Kevin R. Canini, Bongwon Suh, and Peter L. Pirolli. 2011. Finding credible information sources in social networks based on content and social structure. In Proceedings of the IEEE 3rd Inernational Conference on Social Computing (SocialCom) and IEEE 3rd International Conference on Privacy, Security, Risk and Trust (PASSAT). IEEE, 1-8.

[4] Carlos Castillo, Marcelo Mendoza, and Barbara Poblete. 2011. Information credibility on Twitter. In Proceedings of the 20th International Conference on World Wide Web (WWW'11). ACM, New York, NY, 675-684. DOI : https://doi.org/10.1145/1963405.1963500

[5] Carlos Castillo, Marcelo Mendoza, and Barbara Poblete. 2013. Predicting information credibility in time-sensitive social media. Internet Res. 23, 5 (2013), 560-588.

[6] Niall J. Conroy, Victoria L. Rubin, and Yimin Chen. 2015. Automatic deception detection: Methods for finding fake news. Proc. Assoc. Inf. Sci. Technol. 52, 1 (2015), 1-4.

[7] Simon Constable. 2015. "Must-Follow" Twitter Feeds On Markets And Economics. Retrieved from https://www.forbes.com/sites/ simonconstable/2015/08/14/must-follow-twitter-feeds-on-markets-and-economics/\#46f90dc0b09e.

[8] Jared Cummans. 2015. 100 Insightful Futures Traders Worth Following on Twitter. Retrieved from https://commodityhq.com/investorresources/100-insightful-futures-traders-worth-following-on-twitter/.

[9] Daniel Gayo-Avello. 2012. "I wanted to predict elections with Twitter and all I got was this lousy paper"-A balanced survey on election prediction using twitter data. arXiv preprint arXiv:1204.6441 (2012).

[10] Georgios Gravanis, Athena Vakali, Konstantinos Diamantaras, and Panagiotis Karadais. 2019. Behind the cues: A benchmarking study for fake news detection. Exp. Syst. Applic. 128 (2019), 201-213.

[11] Aditi Gupta and Ponnurangam Kumaraguru. 2012. Credibility ranking of tweets during high impact events. In Proceedings of the 1st Workshop on Privacy and Security in Online Social Media. ACM, 2.

[12] Aditi Gupta, Ponnurangam Kumaraguru, Carlos Castillo, and Patrick Meier. 2014. Tweetcred: Real-time credibility assessment of content on Twitter. In Proceedings of the International Conference on Social Informatics. Springer, 228-243.

[13] Manish Gupta, Peixiang Zhao, and Jiawei Han. 2012. Evaluating event credibility on Twitter. In Proceedings of the SIAM International Conference on Data Mining. SIAM, 153-164.

[14] Teng-Chieh Huang, Razieh Nokhbeh Zaeem, and K. Suzanne Barber. 2019. It is an equal failing to trust everybody and to trust nobody: Stock price prediction using trust filters and enhanced user sentiment on twitter. ACM Trans. Internet Technol. 19, 4 (Sept. 2019). DOI : https://doi.org/10.1145/3338855

[15] Shimon Kogan, Tobias J. Moskowitz, and Marina Niessner. 2019. Fake news: Evidence from financial markets. Retrieved from SSRN 3237763 (2019).

[16] Rizki Kurniati and Dwi H. Widyantoro. 2017. Identification of Twitter user credibility using machine learning. In Proceedings of the 5th International Conference on Instrumentation, Communications, Information Technology, and Biomedical Engineering (ICICI-BME'17). IEEE, 282-286.

[17] Sejeong Kwon, Meeyoung Cha, Kyomin Jung, Wei Chen, and Yajun Wang. 2013. Prominent features of rumor propagation in online social media. In Proceedings of the IEEE 13th International Conference on Data Mining. IEEE, 1103-1108.

[18] Andy Liaw, Matthew Wiener, et al. 2002. Classification and regression by randomforest. R. News 2, 3 (2002), 18-22.

[19] G. Lin, R. N. Zaeem, H. Sun, and K. S. Barber. 2017. Trust filter for disease surveillance: Identity. In Proceedings of the Intelligent Systems Conference (IntelliSys'17). 1059-1066. DOI : https://doi.org/10.1109/IntelliSys.2017.8324259

[20] Linette Lopez and Lucinda Shen. 2015. The 129 finance people you have to follow on Twitter. Retrieved from https://www. businessinsider.com/117-finance-people-to-follow-on-twitter-2014-9.

[21] Marcelo Mendoza, Barbara Poblete, and Carlos Castillo. 2010. Twitter under crisis: Can we trust what we RT? In Proceedings of the 1st Workshop on Social Media Analytics. ACM, 71-79.

[22] Liqiang Nie, Xuemeng Song, and Tat-Seng Chua. 2016. Learning from multiple social networks. Synth. Lect. Inf. Conc. Retr. Serv. 8, 2 (2016), 1-118.

[23] Shivam B. Parikh, Saurin R. Khedia, and Pradeep K. Atrey. 2019. A framework to detect fake tweet images on social media. In Proceedings of the IEEE 5th International Conference on Multimedia Big Data (BigMM'19). IEEE, 104-110.

[24] Shivam B. Parikh, Vikram Patil, and Pradeep K. Atrey. 2019. On the origin, proliferation and tone of fake news. In Proceedings of the IEEE Conference on Multimedia Information Processing and Retrieval (MIPR'19). IEEE, 135-140.

[25] Shivam B. Parikh, Vikram Patil, Ravi Makawana, and Pradeep K. Atrey. 2019. Towards impact scoring of fake news. In Proceedings of the IEEE Conference on Multimedia Information Processing and Retrieval (MIPR'19). IEEE, 529-533.

[26] Srijith Ravikumar, Kartik Talamadupula, Raju Balakrishnan, and Subbarao Kambhampati. 2013. RAProp: Ranking tweets by exploiting the tweet/user/web ecosystem and inter-tweet agreement. In Proceedings of the 22nd ACM International Conference on Information \& Knowledge Management. ACM, 2345-2350.

[27] Eduardo J. Ruiz, Vagelis Hristidis, Carlos Castillo, Aristides Gionis, and Alejandro Jaimes. 2012. Correlating financial time series with micro-blogging activity. In Proceedings of the 5th ACM International Conference on Web Search and Data Mining (WSDM'12). ACM, New York, NY, 513-522. DOI : https://doi.org/10.1145/2124295.2124358

[28] Derek Ruths and Jürgen Pfeffer. 2014. Social media for large studies of behavior. Science 346, 6213 (2014), 1063-1064. 
[29] Harald Schoen, Daniel Gayo-Avello, Panagiotis Takis Metaxas, Eni Mustafaraj, Markus Strohmaier, and Peter Gloor. 2013. The power of prediction with social media. Internet Res. 23, 5 (2013), 528-543.

[30] Chengcheng Shao, Giovanni Luca Ciampaglia, Onur Varol, Alessandro Flammini, and Filippo Menczer. 2017. The spread of fake news by social bots. arXiv preprint arXiv:1707.07592 (2017), 96-104.

[31] Karishma Sharma, Feng Qian, He Jiang, Natali Ruchansky, Ming Zhang, and Yan Liu. 2019. Combating fake news: A survey on identification and mitigation techniques. ACM Trans. Intell. Syst. Technol. 10, 3 (2019), 1-42.

[32] Wanita Sherchan, Surya Nepal, and Cecile Paris. 2013. A survey of trust in social networks. ACM Comput. Surv. 45, 4 (Aug. 2013). DOI : https://doi.org/10.1145/2501654.2501661

[33] Kai Shu, Amy Sliva, Suhang Wang, Jiliang Tang, and Huan Liu. 2017. Fake news detection on social media: A data mining perspective. ACM SIGKDD Explor. Newslett. 19, 1 (2017), 22-36.

[34] Kai Shu, Suhang Wang, and Huan Liu. 2019. Beyond news contents: The role of social context for fake news detection. In Proceedings of the 12th ACM International Conference on Web Search and Data Mining. ACM, 312-320.

[35] Yazhe Wang, Jamie Callan, and Baihua Zheng. 2015. Should we use the sample? Analyzing datasets sampled from twitter's stream API. ACM Trans. Web 9, 3 (June 2015). DOI : https://doi.org/10.1145/2746366

[36] Razieh Nokhbeh Zaeem, David Liau, and K. Suzanne Barber. 2018. Predicting disease outbreaks using social media: Finding trustworthy users. In Proceedings of the Future Technologies Conference. Springer, 369-384. DOI : https://doi.org/10.1007/978-3-030-02686-8_29

[37] Xinyi Zhou, Reza Zafarani, Kai Shu, and Huan Liu. 2019. Fake news: Fundamental theories, detection strategies and challenges. In Proceedings of the 12th ACM International Conference on Web Search and Data Mining. 836-837.

Received November 2019; revised October 2020; accepted January 2021

Digital Threats: Research and Practice, Vol. 2, No. 2, Article 17. Publication date: April 2021. 